\title{
Bilateral Double-Pigtail Ureteral Stent Placement for Management of Ureteral Obstruction Secondary to Transitional Cell Carcinoma in a Dog
}

\author{
Monica Carolina Nery Wittmaack, Guilherme Sembenelli, Paola Castro Moraes, \\ Luís Gustavo Gosuen Gonçalves Dias, Paloma Espirito Santo Silva \& Cynthia Bueno Mani
}

\begin{abstract}
Background: Transitional cell carcinoma (TCC) usually affects the trigone region of the bladder and proximal portion of the urethra. TCC in dogs is often complicated by local tumor invasion and obstruction of the urethra, ureters, or both. Urinary obstruction is the cause of death in approximately $60 \%$ of dogs with TCC. Radical surgeries are associated with morbidity and mortality rates. Stents have recently been evaluated for use in dogs with ureteral obstruction resulting from a variety of urinary tract tumors. This report aims to describe bilateral ureteral stent placement for treatment of malignant ureteral obstruction and long-term follow-up in a dog.

Case: An 11-year-old female spayed Maltese dog with ureteral obstruction secondary to transitional cell carcinoma (TCC) in the bladder trigone. After palliative debulking procedure and diagnostic of TCC in bladder and NSAIDs treatment, recurrence has occurred causing ureteral obstruction and TCC had invaded the abdominal wall. Abdominal wall local tumor resection, trigone mass debulking and bilateral ureteral stent placement was made. A double-pigtail ureteral stent of appropriate length was advanced to bypass the ureteral obstruction. Stent sizes were $3.5 \mathrm{Fr}$ in diameter and from 8 to $32 \mathrm{~cm}$ in length. The patient underwent surgical resection of the transitional cell carcinoma in the abdominal wall. The correct location of the bilateral ureteral pigtail stent was certified by abdominal radiography. Recovery was uneventful and the dog was discharged 2 days after surgery. Eleven months after stent placement, the dog developed lumbar vertebrae metastasis, without evidence of recurrent ureteral obstruction. The owners elected euthanasia 517 days after original presentation and 337 days after ureteral stent placement. Euthanasia was unrelated to the local tumor obstruction but was related to the bone metastasis.

Discussion: Ureteral stent placement is feasible techniques for treatment of bladder TCC in dogs, with the objective of delaying the evolution of the disease and preventing ureteral obstruction. According to previous studies, although distant metastatic disease is worrisome, in the majority of dogs with bladder TCC, the primary location of the tumor is the most common cause of death. In patients with ureteral obstruction, early intervention preserves functional renal tissue. Thus, relief of obstruction should be recommended as soon as possible before irreversible renal damage occurs. The authors encourage aggressive and timely intervention, particularly when ureteral obstruction is bilateral. In the present report, 90 days postoperatively local recurrence causing ureteral obstruction was not evidenced on bladder ultrasound images and laboratory test follow-up. After this period, until 180 days after debulking surgery, tumor recurrence occurred, causing clinical signs and compromising renal function. Complications associated with stent placement included stent migration, recurrent ureteral obstruction, stranguria/pollakiuria, presumably due to irritation of the trigonal region from the distal stent; imperfect stent location; ureteral trauma during stent placement; and urinary tract infection. In the present report, the ureteral stents were placed for palliative treatment for malignant ureteral obstructions. Although urinary tract infection was recurrent, other complications associated with bilateral ureteral stent did not occur, such as accidental dislocation or stent obstruction by the tumor. The results obtained were satisfactory for urinary tract obstruction, allowing survival of 517 days. These findings may support long-term ureteral stenting in veterinary patients.
\end{abstract}

Keywords: urothelial carcinoma, neoplasia, soft tissue surgery.

DOI: $10.22456 / 1679-9216.100814$ 


\section{INTRODUCTION}

Transitional cell carcinoma (TCC) is the most common neoplasia of the canine urinary tract and usually a high-grade invasive cancer. Transitional cell carcinoma usually affects the trigone region of the bladder and proximal portion of the urethra. Urinary obstruction is the cause of death in approximately $60 \%$ of dogs with TCC [4].

Treatment options for the management of obstructive tumor include urethral and ureter stents placement, cystostomy tube placement and aggressive surgical interventions. In many cases, owners elect euthanasia. Multiple surgical techniques have been described to manage urinary tract obstruction, including resection of trigone region of the bladder and ureteral reimplantation, radical cystectomy with ureterocolonic anastomosis, cutaneous ureterostomy and others diversion techniques. However, these techniques are technically challenging and are associated with substantial complications, such as metabolic derangements, pyelonephritis, ureteral perforation, recurrent obstruction, ureteral stricture, hydroureter, hydronephrosis, tumor seeding and poor quality of life. Radical surgeries are associated with morbidity and mortality rates [4]. Stents have recently been evaluated for use in dogs with ureteral obstruction resulting from a variety of urinary tract tumors [7].

The purpose of the study reported here was to describe a minimally invasive, potentially long-term treatment option to provide decompression of the renal collection system with the use of double pigtail ureteral stents in dogs with obstructive trigonal malignant carcinoma.

\section{CASE}

An 11-year-old female spayed Maltese dog, $4 \mathrm{~kg}$, with a history of dysuria and intermittent hematuria, was referred for clinical care presenting a history of treatment for lower urinary tract infection two months prior. The patient was submitted to clinical evaluation and laboratory tests (complete blood count, renal and hepatic panel and urinalysis) that were within normal values. On admission, the dog had no abnormal physical examination findings. Abdominal ultrasonography showed diffuse and irregular thickening of the bladder wall more evident in the trigone region, suggesting an inflammatory or neoplastic process.

Therefore, bladder lavage and subsequent cytology of the fluid were performed, and such examination was considered inconclusive. Thoracic radiographs performed on three projections did not show the presence of metastases. Imaging examinations showed no involvement of regional lymph nodes. Surgical intervention was indicated. Cystotomy was performed. The urinary bladder was diffusely thick with an irregular mucosal margin, most notable at the bladder trigone. The mucosa of the bladder in the trigone region was thickening, inflamed and hemorrhages, ureteral obstruction was not detected and both ureters were catheterized (urethral catheter 04) $)^{1}$ through the ureteral orifice (Figure 1A). Without the diagnostic of the neoplastic or nonneoplastic proliferation, surgical remove (debulking) of the irregularity mass involving all the region of the trigone was then chose (Figure 1B).

The material collected was sent to histopathological analysis that was compatible with invasive transitional cell carcinoma. In the postoperative period, drug therapy was instituted with Cephalexin ${ }^{2}$ [30 mg/kg], Ranitidine ${ }^{2}$ [2 mg/kg], Tramadol Hydrochloride $^{2}$ [3 mg/kg], Dipyrone ${ }^{2}[25 \mathrm{mg} / \mathrm{kg}]$ and Firoco$\mathrm{xib}^{3}\left(\right.$ Previcox $\left.^{\circledR}\right)[5 \mathrm{mg} / \mathrm{kg}]$. A course of mitoxantrone was indicated every 3 weeks. However, the owner chose only treatment with COX-2 inhibitors and not to perform additional adjuvant treatments.

The patient was submitted to clinical evaluation, urinary tract ultrasonography and laboratory tests (complete blood count, measurement of BUN and serum creatinine concentrations, urinalysis with bacteriologic culture and susceptibility testing) 2 weeks after the procedure and then every 3 months thereafter.

Six months after surgery, patient had evidence of worsening clinical signs and symptoms of urinary tract disease. Such as hematuria, dysuria and

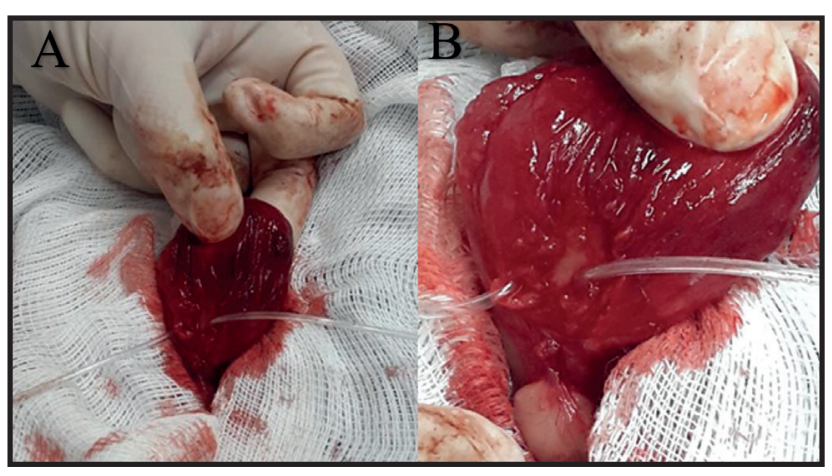

Figure 1. Photograph of the luminal surface of the opened urinary bladder effaced by TCC. A- Intraoperative intravesicular photograph showing diffuse bladder wall thickening with irregular and hemorrhage mucosa, both ureters is catheterized through the ureteral orifice. B- Initial debulking procedure of the irregularity of bladder mucosa in the trigone region. 


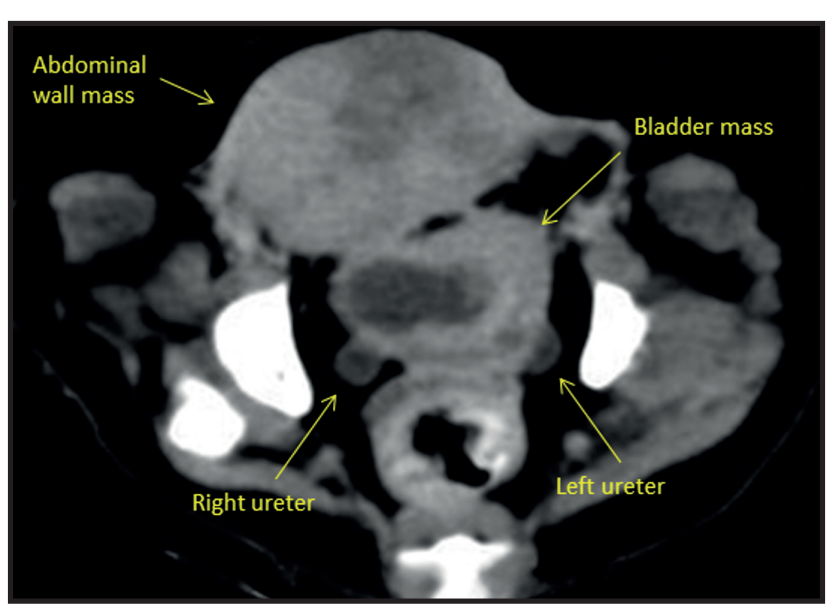

Figure 2. Abdominal computed tomography shows a mass along the urinary bladder involving both ureters in close contact with the mass on the abdominal wall.

pollakiuria. In the physical exam patient had palpable caudal abdominal mass. Patient had evidence of azotemia (BUN concentration $95 \mathrm{mg} / \mathrm{dL}$; creatinine concentration, $2.5 \mathrm{mg} / \mathrm{dL}$ ). Results of bacterial culture of a urine sample were positive, and appropriate antimicrobials were administered for 30 days. Abdominal ultrasound showed a structure in abdominal wall with approximately dimensions of $3.0 \mathrm{~cm}$ x $3.5 \mathrm{~cm}$, which precluded examination of the bladder. Bilateral ureteral obstruction was suspected. Ultrasound image of the bladder demonstrated dilation of the ureters at the trigone level. Tomography was performed. On abdominal $\mathrm{CT}$, a mass was identified in the caudodorsal aspect of the urinary bladder, centered on the trigone region involving both ureters and a caudal mass involving abdominal wall was detected (Figure 2). Both ureters were moderately dilated. There was bilateral hydronephrosis. The mass in the bladder was characterized by a broad-based surface contact, mildly irregular mucosal contour, loss of wall layering and a thickness of $0.8-1.5 \mathrm{~cm}$. The urethra and medial iliac lymph nodes were normal.

On the $180^{\text {th }}$ day, patient had evidence of recurrent neoplasia at the bladder trigone, invasion by tumor on the bilateral side with hydroureter and hydronephrosis, and suspect of the carcinoma seeding in the abdominal wall. Treatment options for management of the ureteral obstruction and progressive disease discussed with the owners included: total cystectomy, ureteral stenting, and palliative quimiotherapy. At this time, surgical intervention was decided: abdominal wall local tumor resection, trigone mass debulking and bilateral ureteral pig tail stent placement. The patient underwent diagnostic imaging prior to stent placement included thoracic tomography and abdominal ultrasonography and had no evidence of metastatic disease. The entire ventral aspect of the abdomen was prepared for aseptic surgery. A ventral median celiotomy incision from $2 \mathrm{~cm}$ caudal to the xiphoid extending to the pubis was made. On exploratory celiotomy, the ureters were bilaterally enlarged. The bladder was grossly abnormal, with a markedly thickened wall and was firm on palpation. Once urinary bladder access was achieved, bladder trigone and obstruction of the ureters at the level of the ureteral papillae was noted. After debulking procedure, the double pig tail and guide wire was then manipulated gently, used to achieve access across the tumor and advanced into the ureter guided cranially towards the kidney. A double-pigtail ureteral stent (Plasti-med $\left.{ }^{\circledR}\right)^{4}$ of appropriate length was advanced to bypass the ureteral obstruction. Stent sizes were $3.5 \mathrm{Fr}$ in diameter and from 8 to $32 \mathrm{~cm}$ in length. Once the proximal end of the stent was advanced to the renal pelvis, the guidewire was slowly withdrawn to allow the pigtail stent to curve into the renal pelvis and trigone area, leaving the double pigtail stent in place (Figure 3). The 2 curves of pig-tail stent ending in the bladder lumen had to be cut for better bladder closure. There was an irregularly shaped circumferential mass in the abdominal wall musculature, the serous surface of the bladder had macroscopic lesions, and the median ligament ventrally close to the pubic bone was involved between these two structures (Figure 4).

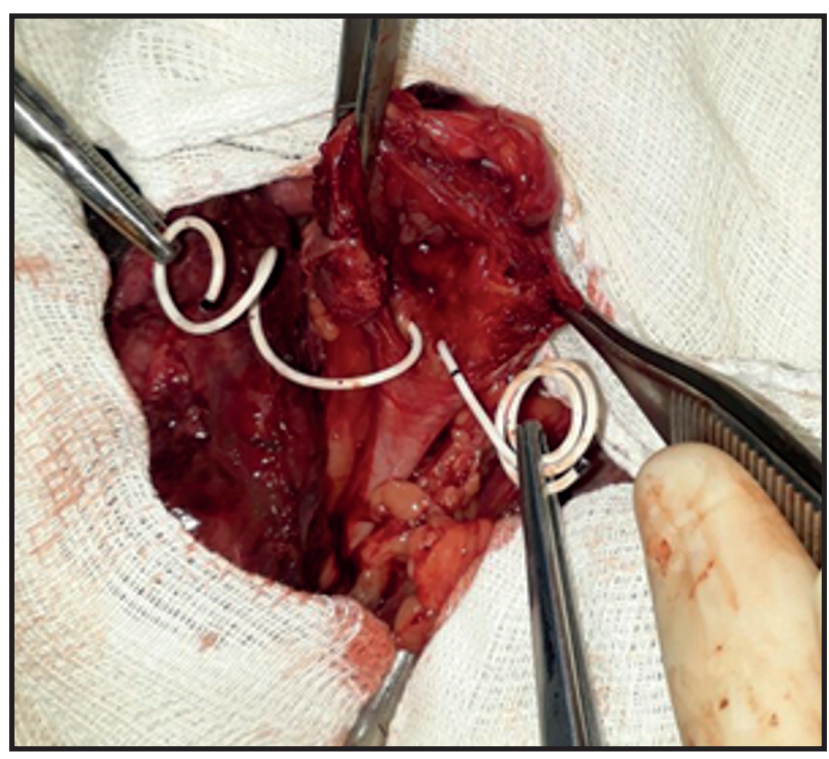

Figura 3. Intraoperative photograph showing the distal part of two doublepigtail ureteral catheter exiting the ureteral opening within the urinary bladder lumen. 


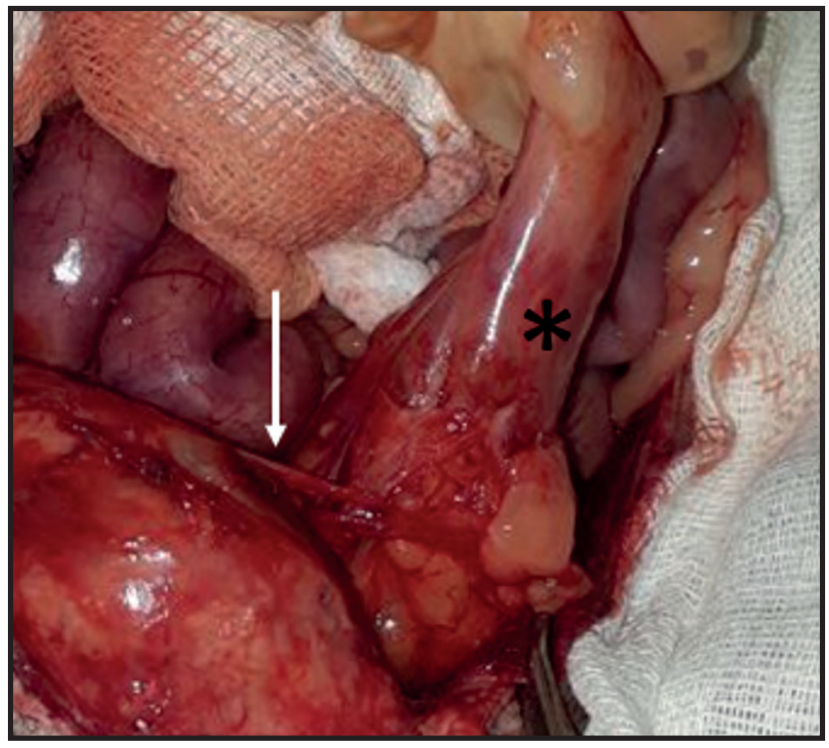

Figure 4. Photographs of tumor of a dog with TCC in the urinary bladder and ABWTCC. Intraoperative photograph showing ventral part of urinary bladder $(*)$, ventral median ligament (white arrow) and mass in the abdominal wall (black arrow).

The round ligament of the bladder was dissected and removed using electrocautery. The patient underwent surgical resection of the transitional cell carcinoma in the abdominal wall. The site was lavaged with sterile saline $^{2}(0.9 \% \mathrm{NaCl})$ solution and the incision closed in layers. The correct location of the bilateral ureteral pigtail stent was certified by abdominal radiography.

The dog recovered in intensive care and was administered IV fluids, fentanyl ${ }^{2}$ [2-6 mg/kg/h], ketamine $^{5}\left[0.1-0.2 \mathrm{mg} / \mathrm{kg} / \mathrm{h}\right.$ as needed], and cefazolin ${ }^{2}$ [22 mg/kg IV every $8 \mathrm{~h}$ ]. Recovery was uneventful and the dog was discharged 2 days after surgery with tramadol$^{2}$ [20 mg orally every $8 \mathrm{~h}$ ], cephalexin ${ }^{2}$ [120 mg orally every $8 \mathrm{~h}]$ and piroxicam ${ }^{6}$ treatment $[0.3 \mathrm{mg} / \mathrm{kg}$ every $24 \mathrm{~h}]$. On histopathology, the mass was definitively diagnosed as a transitional cell carcinoma affecting the entire urinary bladder, with extensive local invasion of the abdominal wall.

The patient presented excellent postoperative recovery and remained stable until 330 days after the second surgical intervention, being monitored monthly for clinical staging. Follow-up ultrasonography was performed and had evidence of improved hydronephrosis and hydroureter, and normalization in BUN and serum creatinine concentrations. Result of bacterial culture of a follow-up urine sample collected after stent placement was positive 2 times, and appropriate antimicrobials were administered for 30 days.

The dog returned 11 months after stent placement for management of ataxia, paraparesis, right limb lameness and muscle atrophy and focal pain on lumbar palpation. At this time, the patient was restaged. During this time, long-term ultrasonography follow-up after stent placement showed slow tumor progression in the bladder and abdominal wall, without evidence of recurrent ureteral obstruction. Radiographic showed alteration in lumbar vertebrae. A metastatic TCC lesion was suspected in lumbar vertebrae and was suspected with enlarged sublumbar lymph nodes (Figure 5). The dog was medicated with hydromorphone ${ }^{2}[0.05 \mathrm{mg} / \mathrm{kg}$ IM] and clinical therapy support was initiated at analgesic support and long term AINES.
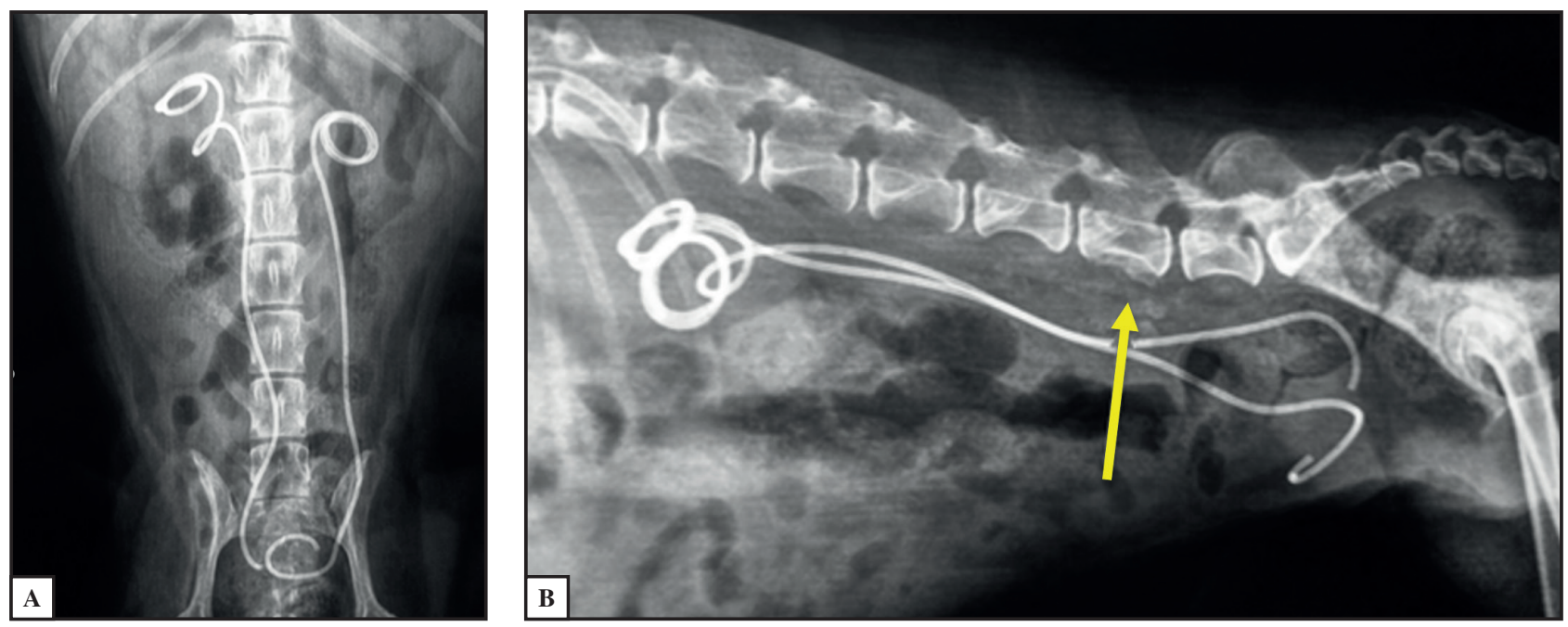

Figure 5. Ventrodorsal and lateral radiographic views of the abdomen of a dog with TCC of the bladder and suspect of metastatic TCC lesion. A- Ventrodorsal radiographic view showing placement of bilateral double-pigtail ureteral stent. B- Laterolateral radiographic view showing soft tissue opacity ventral to lumbar vertebrae displacing the colon ventrally consistent with moderate sublumbar lymph node enlargement, and concurrent moth eaten lysis and new bone at ventral margin of the vertebral body of L6 (arrow), consistent with lymph node and vertebral metastases. 
Progression of signs results in a chronically painful condition. Pain upon direct palpation on lumbar vertebrae was present and painful reaction, aggression toward owners, unwillingness to play, and a general lethargy. Obvious localized pain was found on physical examination. In 510 days after first surgery, the right pelvic limb was positioned in a proprioception deficit, firm and painful pelvic limb muscles; cool, cyanotic nail beds. The dog returned for reassessment 1 week later (17 months from initial admission). The dog was quiet, thin, and her weight had decreased from $4 \mathrm{~kg}$ on initial admission to $2.9 \mathrm{~kg}$, with most this weight loss occurring in the previous 2 months. The owners elected euthanasia 517 days from initial admission (first surgery) and 337 days after ureteral stent placement. Necropsy was declined.

\section{DISCUSSION}

Ureteral stent placement is feasible techniques for treatment of bladder TCC in dogs, with the objective of delaying the evolution of the disease and preventing ureteral obstruction. According to previous studies, although distant metastatic disease is worrisome, in the majority of dogs with bladder TCC, the primary location of the tumor is the most common cause of death. In patients with ureteral obstruction, early intervention preserves functional renal tissue [11]. Thus, relief of obstruction should be recommended as soon as possible before irreversible renal damage occurs [1]. The authors encourage aggressive and timely intervention, particularly when ureteral obstruction is bilateral. In the present report, 90 days postoperatively local recurrence causing ureteral obstruction was not evidenced on bladder ultrasound images and laboratory test follow-up. After this period, until 180 days after debulking surgery, tumor recurrence occurred, causing clinical signs and compromising renal function. Complications associated with stent placement included stent migration, recurrent ureteral obstruction, stranguria/pollakiuria, presumably due to irritation of the trigonal region from the distal stent; imperfect stent location; ureteral trauma during stent placement; and urinary tract infection [1-7]. In the present report, the ureteral stents were placed for palliative treatment for malignant ureteral obstructions. Although urinary tract infection was recurrent, other complications associated with bilateral ureteral stent did not occur, such as accidental dislocation or stent obstruction by the tumor. The results obtained were satisfactory for urinary tract obstruction, allowing survival of 517 days. These findings may support long-term ureteral stenting in veterinary patients.

Local tumor recurrence is a frequent complication after bladder TCC resection. The reasons related to it are presence of residual tumor at the site of resection, neoplastic transformation of the remaining bladder mucosa and implantation of tumor cells into the surgical wound. Incomplete excision is obviously a significant risk factor and macroscopic observation may not be sufficient to delineate the tumor extent. This development is also known as the "cancer field effect" where the entire bladder lining undergoes neoplastic transformation in response to carcinogenic agents. Seeding and intraluminal implantation of tumor cells after surgical manipulation are also related to cancer recurrence [2]. In the case described, debulking was performed for definitive diagnosis, and considering the benefits of non-aggressive surgical resection for certain types of neoplasms, debulking procedure (increased local control) combined with adjuvant therapies, may provide better results in quality of life, corroborating previous study [4].

Total Cystectomy for Treatment of TCC of the Urethra and Bladder Trigone in a Dog was described previously [2]. In this study, after initial treatment for TCC of the bladder trigone with urethral stent placement and chemotherapy, the dog developed urinary incontinence after stent placement, pulmonary metastasis and local progression, leading to bilateral ureteral dilatation. After palliative treatment, total cystectomy was performed and resulted in 92 days survival time after procedure. Total cystectomy for treatment of neoplasia is an uncommon procedure in animal patients. It offers the distinct advantage of long-term local control and the clear disadvantage of urinary incontinence and other causes that compromise quality of life. Therefore, other techniques of total cystectomy and urinary diversion have been suggested, but still, postoperative complications are described [810]. In this study, after diagnosis of bladder TCC, total cystectomy was declined by the owner due to related complications and furthermore the advantage of long-term local tumor control was not believed because of its invasive potential.

Medical treatment is the mainstay for TCC therapy in dogs. Results of numerous medical protocols have been reviewed and compared. Nonsteroidal drugs, 
classically piroxicam, offer a good option for owners wishing to limit or avoid use of other chemotherapeutic agents. Medical therapy is not curative, and the goal is remission or stable disease with a good quality of life. The best results (control of TCC growth in $75 \%$ of dogs with a median survival time of a year) are achieved when dogs receive multiple different treatment protocols over the course of their disease [6]. In the case reported, chemotherapy was initiated only with COX-2 inhibitory anti-inflammatory drugs without association of antineoplastic chemotherapy. These facts may contribute for tumor recurrence and seeding.

TCC has also been reported in the abdominal wall in this case. In this location, the cancer is thought to have been implanted during procedures such as surgery to obtain biopsy specimens, tumor removal, tumor debulking or fine-needle aspiration of TCC in the urinary bladder. Reported mechanisms that could be involved in the growth of TCC in incision sites include direct implantation of tumor cells into the incision area at the time of surgery, the microenvironment in a surgical wound that would support tumor cell proliferation should TCC cells arrive there at surgery or through lymphatic or hematogenous spread, impaired immune response in the perioperative period, and increased vascularity in surgical wounds [2]. In this study, during first surgery, preventive measures to avoid "neoplasm seeding" in neighboring structures and abdominal wall was made. Previous study reports another route of spread of TCC to the abdominal wall. According to the authors [5], TCC spread down the median ligament of the urinary bladder, and the location is typically where the ligament attaches ventrally to the pubic bone. Although lymphatic vessels run along the median ligament, it could not be determined whether the cancer cells were traveling in lymphatic vessels or whether they were following the scaffold provided by the ligament. In this case report, TCC probably has penetrated the serosal surface, spread into the median ligament, and infiltrated the abdominal wall musculature [5].

Prolonged survival of dogs with TCC due to better management of the primary tumor and prevention of urinary tract obstruction might have contributed to an apparent increase in distant metastasis. Metastasis to bone is particularly concerning because the ensuing pain often leads to euthanasia; however, little is known of the frequency, site, or nature of TCC skeletal metastasis [3]. In a retrospective analysis, 17 (9\%) of 188 canine TCC cases had histologically confirmed skeletal metastasis, mainly to the vertebrae. The authors concluded that abdominal radiography would facilitate the detection of these metastatic lesions [9]. In the present report, skeletal metastasis had been suspected based on history, physical examination and had additional radiographic signs vertebrae metastases; however, histologically confirmed skeletal metastases was not possible. CT could be helpful in detecting vertebrae metastases, but the dog owner had requested euthanasia due to declining quality of life.

The findings of the present study suggest that ureteral stent placement was safe, effective, and well tolerated in a patient with malignant ureteral obstruction with benefits regarding the prevention of urinary tract obstruction due to neoplastic growth.

\section{MANUFACTURERS}

${ }^{1}$ Embramed Industria e Comercio de Produtos Hospitalares Ltda. São Paulo, SP, Brazil.

${ }^{2}$ Cristália Produtos Químicos Farmacêuticos Ltda. São Paulo, SP, Brazil.

${ }^{3}$ Merial Saúde Animal Ltda. Paulínia, SP, Brazil.

${ }^{4}$ Plastmed Ltda Epp. Istanbul, Turkey.

${ }^{5}$ Ceva Saúde Animal Ltda. Paulínia, SP, Brazil.

${ }^{6}$ Laboratórios Pfizer Ltda. São Paulo, SP, Brazil.

Declaration of interest. The authors report no conflicts of interest. The authors alone are responsible for the content and writing of the paper.

\section{REFERENCES}

1 Berent A.C., Weisse C., Beal M.W., Brown D.C., Todd K. \& Bagley D. 2011. Use of indwelling, double-pigtail stents for treatment of malignant ureteral obstruction in dogs: 12 cases (2006-2009). Journal of the American Veterinary Medical Association. 238(8): 1017-1025.

2 Boston S. \& Singh A. 2014. Total Cystectomy for Treatment of Transitional Cell Carcinoma of the Urethra and Bladder Trigone in a Dog. Veterinary Surgery. 43(3): 294-300.

3 Charney V.A., Miller M.A., Heng H.G., Weng H.Y. \& Knapp D.W. 2017. Skeletal Metastasis of Canine Urothelial Carcinoma: Pathologic and Computed Tomographic. Veterinary Pathology. 54(3): 380-386. 
M.C.N. Wittmaack, G. Sembenelli, P.C. Moraes, et al. 2020. Bilateral Double-Pigtail Ureteral Stent Placement for Management of Ureteral Obstruction Secondary to Transitional Cell Carcinoma in a Dog. Acta Scientiae Veterinariae. 48(Suppl 1): 522.

4 Fulkerson C.M. \& Knapp D.W. 2015. Management of transitional cell carcinoma of the urinary bladder in dogs: a review. The Veterinary Journal. 205(2): 217-225.

5 Higuchi T., Burcham C.N., Childress M.O., Rohleder J.J., Bonney P.L., Ramos-Vara J.A. \& Knapp D.W. 2013. Characterization and treatment of transitional cell carcinoma of the abdominal wall in dogs: 24 cases (1985-2010). Journal of the American Veterinary Medical Association. 242(4): 499-506.

6 Lipscomb V.J. 2018. Bladder. In: Tobias K.M. \& Johnston S.A. (Eds). Veterinary Surgery: Small Animal. St. Louis: Saunders Elsevier, pp.1978-1993.

7 Mathews K. 2018. Ureters. In: Tobias K.M. \& Johnston S.A. (Eds). Veterinary Surgery: Small Animal. St. Louis: Saunders Elsevier, pp.5954-6002.

8 Mclaughlin M.A. 2011. Complications of Lower Urinary Tract Surgery in Small Animals. Veterinary Clinics of North America. 41(5): 889-913.

9 Norris A.M., Laing E.J., Valli V.E., Withrow S.J., Macy D.W., Ogilvie G.K., Tomlinson J., McCaw D., Pidgeon G. \& Jacobs R.M. 1992. Canine bladder and urethral tumors: a retrospective study of 115 cases (1980-1985). Journal of Veterinary Internal Medicine. 6(3): 145-193.

10 Saeki K., Fujita A., Fujita N., Nakagawa T. \& Nishimura R. 2015. Total cystectomy and subsequent urinary diversion to the prepuce or vagina in dogs with transitional cell carcinoma of the trigone area: A report of 10 cases (2005-2011). Canadian Veterinary Journal. 56(1): 73-80.

11 Salnier-Troff F.G., Busoni V. \& Hamaide A. 2008. A Technique for Resection of Invasive Tumors Involving the Trigone Area of the Bladder in Dogs: Preliminary Results in Two Dogs. Veterinary Surgery. 37(5): $427-437$. 